\title{
Pregnancy outcomes in inflammatory bowel disease patients treated with vedolizumab, anti-TNF or conventional therapy: results of the European CONCEIVE study
}

\author{
Annick Moens ${ }^{1}$ (D) | C. Janneke van der Woude ${ }^{2}$ | Mette Julsgaard ${ }^{3}$ (D) | \\ Evelien Humblet $^{4}$ | Juliette Sheridan ${ }^{5}$ | Daniel C. Baumgart ${ }^{6}$ | \\ Cyrielle Gilletta De Saint-Joseph ${ }^{7}$ | Stéphane Nancey ${ }^{8}$ | Jean-François Rahier ${ }^{9}$ | \\ Peter Bossuyt $^{10}$ | Anneline Cremer ${ }^{11}$ | Sophie Dewit ${ }^{12}$ | Carl Eriksson ${ }^{13}$ (iD | \\ Frank Hoentjen $^{14}$ | Thomas Krause ${ }^{15}$ | Edouard Louis ${ }^{16}$ (i) | Elisabeth Macken ${ }^{17}$ | \\ Zoran Milenkovic $^{18}$ | Jochen Nijs ${ }^{19}$ | Annelies Posen ${ }^{20}$ | Anneleen Van Hootegem ${ }^{21}$ | \\ Wouter Van Moerkercke $^{22}$ | Séverine Vermeire ${ }^{1}$ | Ariella Bar-Gil Shitrit ${ }^{23}$ | \\ Marc Ferrante ${ }^{1}$ (iD)
}

${ }^{1}$ Leuven, Belgium

${ }^{2}$ Rotterdam, The Netherlands

${ }^{3}$ Aarhus, Denmark

${ }^{4}$ Genk, Belgium

${ }^{5}$ Dublin, Ireland

${ }^{6}$ Edmonton, $\mathrm{AB}$, Canada

${ }^{7}$ Toulouse, France

${ }^{8}$ Lyon, France

${ }^{9}$ Yvoir, Belgium

${ }^{10}$ Bonheiden, Belgium

${ }^{11}$ Brussels, Belgium

${ }^{12}$ Overpelt, Belgium

${ }^{13}$ Örebro, Sweden

${ }^{14}$ Nijmegen, The Netherlands

${ }^{15}$ Kassel, Germany

${ }^{16}$ Liège, Belgium

${ }^{17}$ Antwerpen, Belgium

${ }^{18}$ Belgrade, Serbia

${ }^{19}$ int-Truiden, Belgium

${ }^{20}$ Tongeren, Belgium

${ }^{21}$ Brasschaat, Belgium

${ }^{22}$ Kortrijk, Belgium

${ }^{23}$ Jerusalem, Israel

\section{Summary}

Background: Women with inflammatory bowel diseases (IBD) often receive biologicals during pregnancy to maintain disease remission. Data on outcome of vedolizumab-exposed pregnancies (VDZE) are sparse.

Aims: To assess pregnancy and child outcomes of VDZE pregnancies and to compare these results to anti-TNF exposed (TNFE) or both immunomodulatory and biologic unexposed (CON IBD) pregnancies.

Methods: A retrospective multicentre case-control observational study was performed.

Results: VDZE group included 79 pregnancies in 73 IBD women. The TNFE and CON IBD group included 186 pregnancies (162 live births) in 164 IBD women and 184 pregnancies (163 live births) in 155 IBD women, respectively. At conception, cases more often had active disease ([VDZE: $36 \%$ vs TNFE: $17 \%, P=.002$ ] and [VDZE: $36 \%$ vs CON IBD: $24 \%, P=.063]$ ). No significant difference in miscarriage rates were found between groups (VDZE and TNFE: $16 \%$ vs $13 \%, P=.567$; VDZE and CON IBD: $16 \%$ vs $10 \%, P=.216$ ). In live-born infants, median gestational age and birthweight were similar between groups. Median Apgar score at birth was numerically equal. Prematurity was similar in the VDZE group compared to the control groups, even

Annick Moens, C. Janneke van der Woude, Ariella Bar-Gil Shitrit, Marc Ferrante have contributed equally.

Conference presentation: Digital oral presentation at European Crohn's and Colitis Organisation 2019; Plenary session at Belgian Week of Gastroenterology 2019; Poster presentation at Digestive Disease Week 2019.

The Handling Editor for this article was Professor Richard Gearry, and it was accepted for publication after full peer-review.

The complete list of author's affiliation are listed in Appendix 1. 
Correspondence

Dr Marc Ferrante, Herestraat 49, 3000

Leuven, Belgium.

Email: marc.ferrante@uzleuven.be

Funding information

A Bar-Gil Shitrit received a grant from

Takeda and Janssen. S. Vermeire and M.

Ferrante are Senior Clinical Investigators of

the Research Foundation Flanders (FWO),

Belgium. when correcting for disease activity during pregnancy. The frequency of congenital anomalies was comparable between groups as were the percentages of breastfed babies. During the first year of life, no malignancies were reported and infants' infection risk did not significantly differ between groups.

Conclusion: No new safety signal was detected in VDZE pregnancies although larger, prospective studies are required for confirmation

\section{1 | INTRODUCTION}

Inflammatory bowel diseases (IBDs), including Crohn's disease (CD) and ulcerative colitis (UC), are often diagnosed during the fertile period of life. This special situation must be taken into account when selecting an appropriate medical treatment. ${ }^{1}$ Previous studies have shown that disease remission prior to conception and throughout pregnancy is crucial for favourable pregnancy outcomes. ${ }^{2}$ In order to achieve disease remission, patients with IBD are frequently treated with biological therapies such as vedolizumab (VDZ), a monoclonal antibody directed against the $\alpha 4 \beta 7$ integrin-mucosal vascular addressin cell adhesion molecule 1 (MAdCAM-1) interaction in the gut. ${ }^{3-6}$ By blocking this interaction, VDZ inhibits the trafficking of gut-homing lymphocytes. ${ }^{3}$ However, data on the use of VDZ during conception and pregnancy remain sparse..$^{7-11}$ We previously reported several pregnancy and neonatal complications among female IBD patients treated with VDZ in a retrospective Belgian study. In particular, a relatively high number of congenital malformations were reported (three out of 23 live births: hip dysplasia, pulmonary valve stenosis and Hirschsprung's disease). Additionally, the published case of a congenital corpus callosum agenesis anomaly in a healthy volunteer exposed to VDZ gave rise to concerns. ${ }^{7,8}$ Since the Belgian study was too small to draw firm conclusions and underlying active disease was a major confounding factor, a pan-European study was performed to increase the number of VDZ-exposed (VDZE) pregnancies and thus the statistical power. The aim of the current study was to assess pregnancy and child outcomes in a larger cohort of women with IBD treated with VDZ in comparison to pregnancy outcomes in IBD patients treated with anti-tumour necrosis factor (anti-TNF) therapy or not treated with immunomodulators (IMs) nor biologics.

\section{2 | MATERIALS AND METHODS}

\section{1 | Study population}

This was a retrospective multicentre $(n=29)$ observational study, whereby European gastroenterologists were asked to report all VDZE pregnancies between March 2009 and December 2018. Further inclusion criteria were that infants had to be at least 3 months old at the time of inclusion in the study. A subset of this study population was already published in previous studies. ${ }^{8,12,13}$ The control groups of anti-TNF-exposed (TNFE) and IMs and biologics unexposed (CON IBD) pregnancies were prospectively collected in collaboration with two centres with a specialised IBD pregnancy clinic (Erasmus Medical Center, Rotterdam, The Netherlands and Shaare Zedek Medical Centre, Jerusalem, Israel). Both centres were asked to enrol 100 consecutive patients per control group. The aim was to include around 100 VDZE pregnancies and twice as many TNFE and CON IBD pregnancies. Cases and controls were not matched.

\section{2 | Ethical considerations}

Data collection started after ethical approval by the Ethics Committee of the University Hospitals Leuven, Leuven, Belgium (S60985) and where necessary after ethical approval by a local committee for other centres.

\section{3 | Data collection}

Information from VDZE pregnancies was collected through a structured electronic case report form (CRF), adapted from the CRF used in the previous Belgian observational study, ${ }^{8}$ and had to be filled out by the treating gastroenterologist (Figure S1).

Information on the perinatal period included mode of delivery and infants' characteristics and complications (gestational age, sex, birthweight, Apgar scores at minutes 1 and 5, congenital malformations, need for intensive care unit admission, development of allergies, vaccinations [in particular administration of live attenuated vaccines such as Rotavirus, Bacillus Calmette-Guérin or oral polio vaccination], breastfeeding, severe infectious complications and malignancies occurring in the first year of life [date of diagnosis, type of infection or tumour, length of hospitalisation, treatment and outcome]). In addition, we collected maternal characteristics at conception and throughout pregnancy: IBD diagnosis and previous IBD-related medical history, obstetrical history (number of previous pregnancies, miscarriages and elective terminations as well as possible complications and outcomes of previous pregnancies), comorbidities, C-reactive protein level prior to conception and throughout pregnancy, disease activity based on physician global assessment (PGA) as well as patients' recorded disease activity, concomitant use of medication at conception, date of conception, smoking and drinking habits during pregnancy, 
use of folic acid supplementation, dose and duration of VDZ therapy, reason for VDZ discontinuation during pregnancy, other IBD medications during pregnancy (including topical therapy, mesalazine, systemic steroids, IMs, anti-TNF therapy and ustekinumab [UST]), disease activity (PGA and patients' recorded disease activity) and changes in treatment (dose escalation or de-escalation, change in time interval or initiation of other treatments) per trimester of pregnancy and complications such as placenta praevia, (pre)-eclampsia, infections during pregnancy, intrauterine growth retardation (IUGR), foetal loss or elective termination and premature rupture of the membranes (PROM).

To avoid missing data, gastroenterologists were requested to contact the patients, general practitioners, paediatricians or other colleagues.

Control centres were asked to contribute the same maternal and neonatal information. However, since these patients were already included in a prospective database prior to this study, not all variables were available.

\subsection{Outcomes}

The aims of this study were (a) to assess the outcome of VDZE pregnancies both for the mother and the child and (b) to compare these results to outcomes of TNFE and CON IBD pregnancies. Primary and secondary outcome parameters included the number of live births, stillbirths, miscarriages and elective terminations, preterm deliveries, infections during pregnancy (including chorioamnionitis), (pre-) eclampsia, placenta praevia, IBD flare during pregnancy and neonatal complications such as IUGR, low birthweight, small for gestational age, low Apgar score at birth, congenital anomalies, admission to neonatal intensive care unit, serious infection or malignancy in the first year of life or at last follow-up.

\subsection{Definitions}

Stillbirth was defined as foetal loss after 20 weeks post-conception. ${ }^{14}$ Preterm delivery was defined as any delivery before 37 weeks of gestation. ${ }^{15}$ Low birthweight was defined as a birthweight less than $2500 \mathrm{~g}$ in a child born at term. ${ }^{15}$ Small for gestational age was defined as a weight below the 10th percentile with regard to gestational age ${ }^{16}$ and a low Apgar score was defined as a score $<7$ at minute $5 .{ }^{17}$ The first trimester was characterised as the time from conception until week 14 of gestation, the second trimester from week 14 to 28 and the third trimester from week 28 until delivery. Severe infectious complications in the newborn were regarded as infectious complications requiring or prolonging hospitalisation. IBD disease activity was retrospectively assessed at conception and during each trimester of pregnancy using PGA and patients' recorded disease activity. PGA options correspond to "active disease" or "remission" and were based on the clinical judgement of the treating gastroenterologist. Clinical remission for UC patients based on patients' recorded disease activity was based on patient-reported outcome (PRO2) scores and defined as a rectal bleeding score of 0 and an absolute number of stools $\leq 3$, and for $C D$ patients it was based on an abdominal pain score $\leq 1$ and liquid to very soft stool frequency $\leq 1.5$ for $C D .{ }^{18}$ Due to a lot of missing patients' recorded scores in the VDZE group and the absence of these scores in the control groups, only PGA was used.

\subsection{Statistical analysis}

Descriptive statistics were used to display the results. For discrete data, proportions and percentages were reported, whereas for continuous data medians with interquartile ranges (IQR) were presented. To compare cases and controls, we used Fisher's exact test or Chi-squared for categorical data and Mann-Whitney $U$ test for continuous data. In case of missing data, these data were not included in the analyses. IBM SPSS Statistics 25.0 software package was used. A $P<.05$ was regarded as statistically significant.

\section{3 | RESULTS}

\section{1 | Study population}

In total, 29 centres from nine countries (Belgium, Denmark, France, Germany, Ireland, Israel, Serbia, Sweden and The Netherlands) reported 79 VDZE pregnancies in 73 unique women resulting in 64 live births including three dizygotic twins. TNFE group included 186 pregnancies in 164 women leading to 162 live births including one pair of twins. CON IBD group included 184 pregnancies in 155 women leading to 163 live births including one pair of twins.

\section{2 | Maternal baseline characteristics}

Baseline characteristics are displayed in Table 1. Groups were comparable regarding age at conception and duration of underlying IBD, although the TNFE group included significantly more women with CD compared to the VDZE group ( $83 \%$ vs $55 \%, P=.0001$ ). At conception, 27 (36\%) VDZE women had clinically active disease according to PGA, 16 (20\%) were on concomitant steroids and 14 (18\%) on IMs. In addition, 42 (55\%) previously failed two other biological treatments. Disease activity at conception and being on steroids were both significantly increased among the VDZE group compared to the TNFE (activity disease: $36 \%$ vs $17 \%, P=.002$; steroid treatment: $20 \%$ vs $8 \%, P=.005$ ) and the CON IBD groups (activity disease: $36 \%$ vs $24 \%, P=.063$, and steroid treatment: $20 \%$ vs $14 \%$, $P=.195)$. The proportion of women who smoked or drank alcohol in pregnancy was comparable between groups, but the intake of folic acid supplementation was higher, though not significant, in the VDZE group compared to the TNFE group and CON IBD group.

\subsection{Medication use in pregnancy}

\subsection{1 | VDZE group $(\mathrm{n}=79)$}

At conception, the median duration of VDZ therapy was 7 (IQR 3-12) months and $83 \%$ of VDZ-treated patients were on maintenance 
TAB LE 1 Baseline maternal characteristics $(n=392)$

\begin{tabular}{|c|c|c|c|c|c|}
\hline & $\begin{array}{l}\text { VDZE group } \\
(n=73)\end{array}$ & $\begin{array}{l}\text { TNFE group } \\
(\mathrm{n}=164)\end{array}$ & $\begin{array}{l}\text { CON IBD } \\
\text { group }(n=155)\end{array}$ & $\begin{array}{l}P \text {-value (VDZE } \\
\text { vs TNFE) }\end{array}$ & $\begin{array}{l}P \text {-value (VDZE vs } \\
\text { CON IBD) }\end{array}$ \\
\hline Crohn's disease (\%) & $40 / 73(55)$ & 136/164 (83) & $86 / 155(55)$ & $0.0001^{*}$ & 0.566 \\
\hline $\begin{array}{l}\text { Median (IQR) duration of disease at } \\
\text { conception (y) }\end{array}$ & $7(5-10)$ & $7(4-12)$ & $6(3-11)$ & 0.754 & 0.363 \\
\hline $\begin{array}{l}\text { Median (IQR) C-reactive protein before } \\
\text { conception (mg/L) }\end{array}$ & $6.7(2.3-14)$ & NA & NA & NA & NA \\
\hline \multicolumn{6}{|l|}{ Usage during pregnancy (\%) } \\
\hline Smoking & $4 / 79(5)$ & $12 / 186(6)$ & $8 / 184(4)$ & 0.282 & 0.299 \\
\hline Alcohol use & $2 / 79(3)$ & $1 / 185(0.5)$ & $1 / 183(0.5)$ & 0.214 & 0.217 \\
\hline Folic acid supplements & $76 / 78(97)$ & $165 / 182(91)$ & $164 / 181(91)$ & 0.068 & 0.068 \\
\hline \multicolumn{6}{|l|}{ Parity (\%) } \\
\hline \multicolumn{6}{|c|}{ Clinically active disease during pregnancy (PGA, \%) } \\
\hline Conception & $27 / 74(36)$ & $31 / 182(17)$ & $43 / 179(24)$ & $0.002^{*}$ & 0.063 \\
\hline First trimester & $23 / 75(31)$ & 26/138 (19) & $25 / 113(22)$ & 0.061 & 0.232 \\
\hline Second trimester & $15 / 61(25)$ & $19 / 125(15)$ & $30 / 119(25)$ & 0.157 & 1.000 \\
\hline Third trimester & $11 / 60(18)$ & $16 / 121(13)$ & $29 / 115(25)$ & 0.381 & 0.347 \\
\hline \multicolumn{6}{|l|}{ Previous number of biologicals (\%) } \\
\hline 0 & $5 / 77(6)$ & NA & NA & NA & NA \\
\hline 1 & $22 / 77(29)$ & & & & \\
\hline 2 & $42 / 77(55)$ & & & & \\
\hline E2 & $17 / 32(53)$ & $9 / 28(32)$ & $39 / 68(57)$ & 0.123 & 0.829 \\
\hline E3 & $13 / 32(41)$ & $17 / 28(61)$ & $14 / 68(21)$ & 0.195 & 0.053 \\
\hline \multicolumn{6}{|l|}{ Disease location CD (\%) } \\
\hline L1 & $5 / 40(13)$ & $56 / 136(41)$ & $29 / 86(34)$ & $0.0006^{*}$ & $0.017^{*}$ \\
\hline L2 & $4 / 40(10)$ & $19 / 136(14)$ & $20 / 86(23)$ & 0.604 & 0.092 \\
\hline L3 & $31 / 40(78)$ & $60 / 136(44)$ & $37 / 86(43)$ & $0.0003^{*}$ & $0.0005^{*}$ \\
\hline L4 & $2 / 40(5)$ & $1 / 136(0.7)$ & 0 & 0.130 & 0.099 \\
\hline \multicolumn{6}{|l|}{ Disease behaviour CD (\%) } \\
\hline B1 & $17 / 40(43)$ & $71 / 136(52)$ & $61 / 86(71)$ & 0.369 & $0.003^{*}$ \\
\hline B2 & $9 / 40(23)$ & $21 / 136(15)$ & $7 / 86(8)$ & 0.340 & $0.041^{*}$ \\
\hline B3 & $14 / 40(35)$ & $44 / 136(32)$ & $18 / 86(21)$ & 0.849 & 0.123 \\
\hline Perianal disease CD (\%) & $17 / 40(43)$ & $36 / 135(27)$ & $10 / 86(12)$ & 0.077 & $0.0001^{*}$ \\
\hline Prior surgery for IBD (\%) & $18 / 73(25)$ & $64 / 164$ (39) & $22 / 155(14)$ & $0.038^{*}$ & 0.063 \\
\hline
\end{tabular}

Abbreviations: CD, Crohn's disease; CON IBD, immunomodulatory or biologic unexposed pregnancies; IBD, inflammatory bowel disease; IMs, immunomodulators; IQR, interquartile range; PGA, physician global assessment; TNFE, anti-TNF-exposed pregnancies; VDZE, vedolizumab exposed pregnancies; UC, ulcerative colitis.

Fixed variables were determined at the time of the first pregnancy (eg diagnosis CD or UC), while adjustable variables were determined for each pregnancy (eg disease activity).

${ }^{*}$ means a $P$-value of $<0.05$ and thus a statistical signifcant result. 
therapy with 300-mg intravenous VDZ every 4 weeks (Q4W, $\mathrm{n}=21$, $26 \%$ ) or every 8 weeks (Q8W, $n=45,57 \%$ ). At gestational week 15, one of these patients underwent dose de-escalation (Q4W-Q8W) and at gestational week 20 , another woman needed dose escalation (Q8W-Q4W). Ten patients (13\%) received induction therapy at conception and three (4\%) initiated VDZ treatment after conception. The latter included two patients who initiated VDZ treatment at gestational week 7 , one in combination with mesalazine throughout pregnancy and one with corticosteroids for one trimester, after loss of response to adalimumab (ADM) and golimumab respectively. A third patient initiated VDZ treatment at gestational week 17 due to flare and this in combination with corticosteroids throughout pregnancy. During pregnancy, the median number of administered VDZ infusions was 4 (IQR 2-5). For women with live born children, $(n=38,62 \%)$ discontinued VDZ treatment in pregnancy, equivalent to $13(20 \%)$ in the first, 21 (34\%) in the second and 5 (8\%) in the third trimester. Twentythree (38\%) women continued VDZ therapy throughout pregnancy. The reasons for stopping VDZ treatment were: advice of multidisciplinary consultation mainly because of unknown effects ( $n=31,78 \%$ ), at the request of the patient ( $n=3,8 \%$ ), upon recommendation of the treating gastroenterologist $(n=2,5 \%$ ) or gynaecologist $(n=1,2 \%)$, due to loss of response and need for colectomy $(n=1,2 \%)$ and in two cases no reasoning was available (5\%). After delivery, 34 (76\%) women restarted VDZ treatment, two (4\%) switched to anti-TNF therapy, three (7\%) to UST, four (9\%) patients did not restart therapy and another two (4\%) received other treatments. The use of VDZ at conception and during pregnancy is shown in Figure 1, whilst Figure 2 displays the use of concomitant medication during pregnancy.

\subsubsection{TNFE group $(n=186)$}

At conception, 84 (45\%) were on infliximab (IFX) monotherapy, 101 (54\%) on ADM monotherapy and the remaining patient (0.5\%) initiated $A D M$ treatment during the first trimester. In patients receiving IFX monotherapy, the following schedules were administered: $5 \mathrm{mg} /$ kg every $4(n=3,4 \%)$, every $6(n=16,19 \%)$, every $7(n=7,8 \%)$, every $8(n=48,57 \%)$ or every $9(n=2,2 \%)$ weeks, two $(2 \%)$ patients received $10 \mathrm{mg} / \mathrm{kg}$ every 6 or 8 weeks and six (7\%) patients had an unknown treatment schedule. Twelve women (20\%) discontinued their IFX treatment during the first trimester, 24 (39\%) during the second trimester and eight (13\%) during the third trimester. Patients who received their last IFX infusion before gestational week 32 were considered as discontinuing IFX therapy in pregnancy. Seventeen (28\%) patients continued IFX treatment throughout pregnancy.

Treatment regimens for ADM included the following: $40 \mathrm{mg} / \mathrm{kg}$ every other week ( $n=71,70 \%$ ), every week ( $n=25,25 \%$ ) or unknown $(n=5,5 \%)$. ADM was discontinued in the first, second or third trimester in six (8\%), $23(32 \%)$ and $13(18 \%)$ patients respectively. Patients receiving their last ADM injection before gestational week 36 were considered as discontinuing ADM therapy in pregnancy. The remaining 17 (23\%) patients received ADM treatment throughout pregnancy.

Of note, the most frequent reason for discontinuing anti-TNF therapy in pregnancy was disease remission. Usage of concomitant therapy is shown in Figure 3.

\subsection{3 | CON IBD group}

The CON IBD group consisted of pregnancies that were not exposed to biologics nor to immunomodulatory drugs. At conception, 92 (50\%) females were being treated with mesalazine monotherapy, 12 (7\%) with monotherapy of steroids, 12 (7\%) with a combination of mesalazine and steroids and the remaining 67 patients (36\%) did not receive any IBD therapy. Of the 24 (13\%) women on steroids, $21(88 \%)$ continued this therapy during the course of pregnancy.

\section{4 | Pregnancy outcomes and complications}

In the VDZE group, $68 \%$ of women $(n=54)$ had been pregnant before and $41 \%(n=22)$ of these previous pregnancies were complicated by PROM ( $n=3,14 \%)$, miscarriage ( $n=14,64 \%)$, ectopic pregnancy ( $n=1,4 \%)$, IBD flare $(n=2,9 \%)$ or other complications $(n=2,9 \%)$. In the TNFE group, 68 women (42\%) had a prior pregnancy of which 29 (43\%) were complicated: 11 (38\%) previous miscarriages, one previous stillbirth (3\%) and 17 (59\%) unknown complication. Sixty-six
FIGURE 1 Use of vedolizumab throughout pregnancy. Trimester 1 : exclusion of 12 early miscarriages and three elective terminations. Trimester 2: exclusion of one late miscarriages, one elective terminations and one stillbirth. Q4W, vedolizumab $300 \mathrm{mg}$ every $4 \mathrm{wk}$; Q8W, vedolizumab $300 \mathrm{mg}$ every $8 \mathrm{wk}$; VDZ, vedolizumab

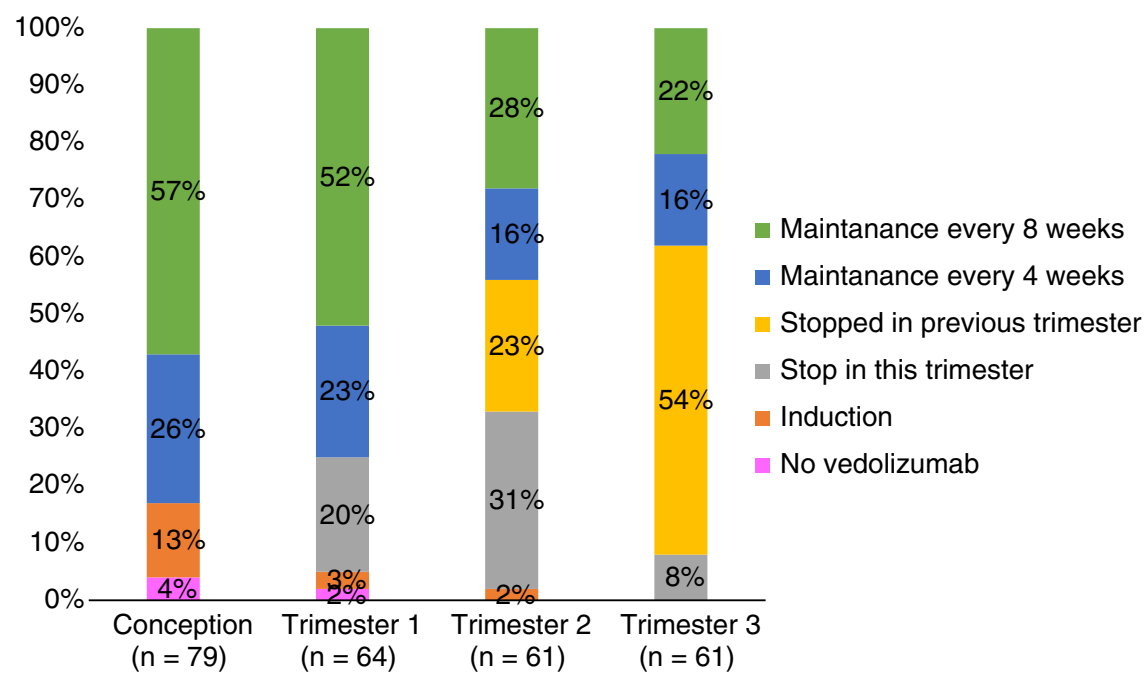



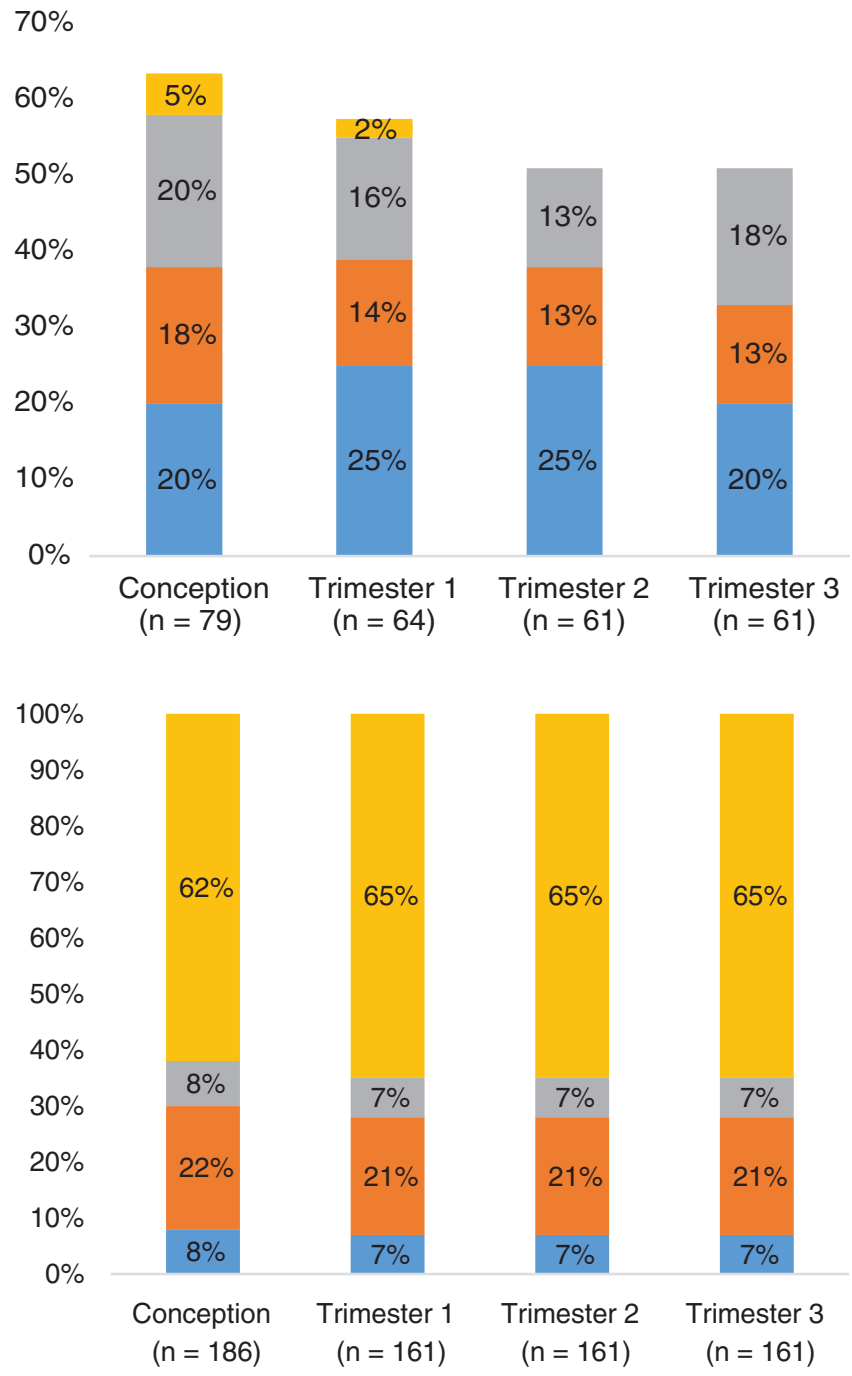

females (43\%) in the CON IBD group had a previous pregnancy and 21 (32\%) of these pregnancies were complicated: eight (38\%) previous miscarriages, five (24\%) previous stillbirths and eight (38\%) unknown complications.

The rate of live-born infants was high (VDZE: 78\%, TNFE: $87 \%$ and CON IBD: $89 \%$ ), not significantly different between the VDZE and TNFE groups ( $P=.103$ ), though significantly higher in the CON IBD group compared to the VDZE group $(P=.038)$ (Figure S2). Excluding patients with active disease at any point in pregnancy resulted in similar rates of live born children in the VDZE group compared to the TNFE ( $82 \%$ vs $83 \%, P=.816)$ and the CON IBD ( $82 \%$ vs $85 \%, P=.617$ ) groups respectively.

In addition, there was no difference in miscarriage rates between the VDZE and TNFE groups ( $16 \%$ vs $13 \%, P=.567$ ) nor between VDZE and CON IBD groups ( $16 \%$ vs $10 \%, P=.216$ ). The proportion of elective terminations was significantly higher in cases compared to controls: VDZE vs TNFE ( $5 \%$ vs $0 \%, P=.007)$ and CON IBD $(5 \%$ vs $0.5 \%, P=.030)$. Overall, the number of stillbirths was low and did not differ between the VDZE and TNFE groups ( $1 \%$ vs $0 \%, P=.298$ ) nor the VDZE and CON IBD groups ( $1 \%$ vs $0 \%, P=.300$ ). When looking only at women with disease remission throughout pregnancy, no

FIGURE 2 Use of concomitant medication in vedolizumab-exposed pregnancies. Trimester 1: exclusion of 12 early miscarriages and three elective terminations. Trimester 2: exclusion of one late miscarriages, one elective terminations and one stillbirth. Patients at conception on anti-TNF therapy where not yet on VDZ therapy

Anti-TNF therapy -Systemic steroids - Immunomodulators -Mesalazine
FIGURE 3 Use of concomitant medication in anti-tumour necrosis factorexposed pregnancies. Trimester 1-3: exclusion of 24 early miscarriages and one elective terminations

\author{
None \\ Mesalazine \\ - Immunomodulators \\ - Systemic steroids
}

difference in the amount of miscarriages was seen in the controls compared to cases (VDZE vs TNFE [16\% vs $17 \%, P=1.000]$; VDZE vs CON IBD [16\% vs $15 \%, P=.799]$, Figure S3).

Other complications in the VDZE group include PROM $(n=3$, all occurred before GW37), IUGR ( $n=2)$, pre-eclampsia $(n=3)$, placenta praevia ( $n=1)$, maternal catheter-related sepsis $(n=1)$ and intraventricular neonatal bleeding $(n=1)$.

\section{5 | Infants' baseline characteristics and neonatal complications}

Neonatal outcomes are displayed in Table 2. In live-born infants, median gestational age and birthweight were similar between groups. Median Apgar score at birth was also numerically equal in all groups. The number of premature born infants was similar in the VDZE group compared to the TNFE (16\% vs $9 \%, P=.151)$ and CON IBD groups (16\% vs $7 \%, P=.079$ ), even after correcting for disease activity in pregnancy (VDZE: $8 \%$ vs TNFE: $4 \%, P=.363$; VDZE: $8 \%$ vs CON IBD: $6 \%, P=.687$ ) (Figure S4). The amount of reported congenital anomalies in live-born children was comparable between groups (VDZE: 5\% vs TNFE: $2 \%, P=.407$; VDZE: $5 \%$ vs CON IBD: $2 \%, P=.354$ ) (Table 3 ). 
In the TNFE group, one case of Down syndrome was reported, but this pregnancy was terminated electively. The percentages of breastfed babies (VDZE: $62 \%$ vs TNFE: $60 \%, P=.877$; VDZE: $62 \%$ vs CON IBD: $64 \%, P=.875)$ were similar in all groups. Regarding vaccination, information was only available for the VDZE group in which all children (for whom information was available, $n=60$ ) followed the national immunisation programme with the exception of the administration of live attenuated vaccines. The latter were given in 17 (28\%) of these infants and include Rotavirus ( $n=14$ ) and/or Bacillus Calmette-Guérin vaccine $(n=3)$. No adverse reactions to vaccinations were reported. During the first year of life, no malignancies were reported and the infants' infection risk did not significantly differ between groups (VDZE: $5 \%$ vs TNFE: $10 \%, P=.326$; VDZE: $5 \%$ vs CON IBD: $12 \%, P=.193)$. Table 4 summarises the reported serious infections in each group.

\section{4 | DISCUSSION}

This is the largest study to date to report on pregnancy outcomes in IBD patients treated with VDZ. Furthermore, it is the first study to compare these results to pregnancy outcomes of women treated with anti-TNF therapy or without IM/biological therapy.

The pregnancy outcomes of our study were favourable with an overall high rate of live births in all groups, though the number of live born infants was significantly higher in the CON IBD group compared to the VDZE group. This is probably due to the higher number of patients with active disease in the VDZE group since the difference in live born infants between both groups disappeared after excluding patients with active disease in pregnancy. Since MAdCAM-1 is expressed in the human placenta during the first trimester of pregnancy, ${ }^{19}$ concerns have been raised about a theoretical risk of early miscarriage with the use of VDZ during conception and pregnancy. However, in this cohort of 79 VDZ-exposed pregnancies, there was no significant difference in the number of miscarriages between the VDZE group and the TNFE or CON IBD group. Furthermore, the percentage of stillbirths was low in all groups.

No dissimilarities were observed between groups with respect to infants' baseline characteristics. Concerning the vaccination status of infants, no comparison could be made due to lack of data from the control patients. The study revealed a high rate of vaccination in the VDZE infants. Almost one-third of babies received one or more live attenuated vaccine of whom seven children were exposed to VDZ until the first trimester, nine until the second trimester and two until the 30th week of gestation. No adverse events after vaccination were reported. The rate of vaccination in our cohort is comparable to previous cohorts of TNFE children, though the administration rate of live attenuated vaccines was lower in our cohort. ${ }^{20-23}$

Overall, the risk of adverse pregnancy outcome was similar within the three groups. Furthermore, the risk of congenital anomalies was low, and comparable among the three groups. The low risk of adverse pregnancy outcome in the present study is in line with previous studies among TNFE and nonbiological therapy-exposed infants born by mothers with IBD. 7,20,24,25

The present study verifies the findings of a large prospective Dutch study that remission in IBD prior to conception and throughout pregnancy is the most import factor to achieve favourable pregnancy outcomes, irrespective of the IBD medication prescribed. ${ }^{26}$ As such, focus should remain on the preconception and pregnancy counselling.

We acknowledge several limitations of the current study. First, the VDZE cases and controls (TNFE and CON IBD) were not entirely

TAB LE 2 Baseline characteristics live-born children $(n=389)^{a}$

\begin{tabular}{|c|c|c|c|c|c|}
\hline & $\begin{array}{l}\text { VDZE group } \\
(\mathrm{n}=64)\end{array}$ & $\begin{array}{l}\text { TNFE group } \\
(\mathrm{n}=162)\end{array}$ & $\begin{array}{l}\text { CON IBD group } \\
(n=163)\end{array}$ & $\begin{array}{l}\text { P-value (VDZE vs } \\
\text { TNFE) }\end{array}$ & $\begin{array}{l}P \text {-value (VDZE } \\
\text { vs CON IBD) }\end{array}$ \\
\hline Gender (F) (\%) & $39 / 64(61)$ & $86 / 144(60)$ & $77 / 150(51)$ & 1.000 & 0.231 \\
\hline Median (IQR) gestational age (wk) & $39(38-40)$ & $39(38-40)$ & $39(38-40)$ & 0.084 & 0.433 \\
\hline Median (IQR) Apgar score at birth & $9(9-10)$ & $9(9-9)$ & $9(9-9)$ & $0.001^{*}$ & $0.002^{*}$ \\
\hline Median (IQR) birthweight (grams) & $\begin{array}{l}3290 \\
(2848-3563)\end{array}$ & $3215(2835-3555)$ & $\begin{array}{l}3237 \\
(2867-3500)\end{array}$ & 0.786 & 0.709 \\
\hline Premature born children (\%) & $10 / 64(16)$ & $14 / 162(9)$ & $12 / 163(7)$ & 0.151 & 0.079 \\
\hline Small for gestational age (\%) & $2 / 64(3)$ & $2 / 162(1)$ & $3 / 163(2)$ & 0.318 & 0.622 \\
\hline Breastfeeding (\%) & $39 / 63(62)$ & $85 / 142(60)$ & $88 / 138(64)$ & 0.877 & 0.875 \\
\hline Congenital anomalies (\%) & $3 / 64(5)$ & $4 / 162(2)$ & $3 / 163(2)$ & 0.407 & 0.354 \\
\hline Infections during the first year of life (\%) & $3 / 64(5)$ & $7 / 67(10)$ & $7 / 59$ (12) & 0.326 & 0.193 \\
\hline $\begin{array}{l}\text { Malignancies during the first year of life } \\
\text { (\%) }\end{array}$ & 0 & 0 & 0 & NA & NA \\
\hline
\end{tabular}

Note: Fisher's exact test used for categorical data and Mann-Whitney $U$ test for continuous data.

Abbreviations: CON IBD: immunomodulatory and biologic unexposed; F: female; IQR: interquartile range; TNFE: anti-TNF-exposed; VDZE: vedolizumab exposed.

${ }^{a}$ Only in children born alive. All preterm deliveries occurred between gestational week 33 and 36 .

*means a $P$-value of $<0.05$ and thus a statistical signifcant result. 
TABLE 3 Congenital anomalies ${ }^{\mathrm{a}}$

\begin{tabular}{|llll|} 
& $\begin{array}{l}\text { VDZE } \\
\text { group } \\
(\mathbf{n}=3)\end{array}$ & $\begin{array}{l}\text { TNFE } \\
\text { group } \\
(\mathbf{n}=4)\end{array}$ & $\begin{array}{l}\text { CON IBD } \\
\text { group } \\
(\mathbf{n}=3)\end{array}$ \\
\hline $\begin{array}{l}\text { Congenital pulmonary valve } \\
\text { stenosis }\end{array}$ & 1 & & \\
\hline $\begin{array}{l}\text { Developmental disability e } \\
\text { causa ignota }\end{array}$ & 1 & \\
\hline $\begin{array}{l}\text { Dilated right ventricle } \\
\text { Hip dysplasia }\end{array}$ & 1 & & 1 \\
\hline Hirschsprung's disease & 1 & 1 & 1 \\
\hline Hypospadias & & 1 & 1 \\
\hline Polydactyly & & $1^{\circ}$ \\
\hline Syndactyly & & 1 & \\
\hline
\end{tabular}

Abbreviations: CON IBD: immunomodulatory and biologic unexposed; TNFE: anti-TNF-exposed; VDZE: vedolizumab exposed. ${ }^{a}$ Only in children born alive. Child was exposed to methotrexate at conception.

TABLE 4 Serious infections ${ }^{\mathrm{a}}$

\begin{tabular}{|llll|}
\hline & $\begin{array}{l}\text { VDZE } \\
\text { group } \\
(\mathbf{n}=3)\end{array}$ & $\begin{array}{l}\text { TNFE } \\
\text { group } \\
(\mathbf{n}=7)\end{array}$ & $\begin{array}{l}\text { CON IBD } \\
\text { group } \\
(\mathbf{n}=7)\end{array}$ \\
\hline Acute otitis media & & 1 & \\
\hline Fever of unknown origin & 1 & 1 & 1 \\
\hline Kawasaki's disease & 1 & & \\
\hline Pyelonephritis & 1 & & \\
\hline Respiratory tract infection & & 1 & 1 \\
\hline Unknown & & 2 & 5 \\
\hline Urinary tract infection & & 2 & \\
\hline
\end{tabular}

Abbreviations: CON IBD, immunomodulatory and biologic unexposed; TNFE, anti-TNF-exposed; VDZE, vedolizumab exposed.

aOnly children born alive and defined as infections for which the child required hospitalisation.

comparable. The VDZE group was more refractory than the TNFE and the CON IBD groups. More than half of the VDZE group had previously been treated with two other biologicals. Furthermore, at conception, the likelihood of active disease and steroid use was increased in the VDZE population. Moreover, among VDZE CD patients, extensive disease, stricturing or fistulising phenotype and perianal involvement were more frequent. Finally, the two control groups (TNFE and CON IBD) were collected in two independent centres with a specialised IBD pregnancy clinic that includes standardised preconception consultation and pregnancy monitoring, whereas the follow-up of VDZE pregnancies varied according to standard clinical practice at each participating centre. Overall, the variation in underlying disease severity and differences in recruitment policy may have affected our relative estimates. ${ }^{27}$ Second, information on VDZE pregnancies was retrospectively acquired, therefore a certain degree of selection bias cannot be excluded. On the other hand, data on controls were prospectively attained prior to the present study resulting in some variables being absent. Last, disease activity was based on PGA as collection of disease activity scores such as PRO2 was incomplete and could therefore not be used to confirm disease activity.

\section{5 | CONCLUSION}

This is the largest European study to date to report on pregnancy outcomes in VDZ-treated IBD patients and to compare these results with women treated either with anti-TNF therapy or without IM and biological therapy. The risk of adverse pregnancy outcome was low in all three groups. After excluding patients with reported active disease at any point in pregnancy, the number of miscarriages was similar in all groups. In addition, the use of VDZ could not be associated with an increased risk of major congenital anomalies in the offspring. The use of VDZ during conception and pregnancy seems to convey a low risk, although this still needs to be confirmed by large, prospective trials.

\section{ACKNOWLEDGEMENTS}

We thank Jens Kjeldsen (Department of Gastroenterology, Odense University Hospital, Odense, Denmark), Lone Larsen (Department of Gastroenterology, Aalborg University Hospital, Aalborg, Denmark), Signe Wildt (Medical Department, Zealand University Hospital, Køge, Denmark), Petra Weimers (Department of Gastroenterology, North Zealand University Hospital, Frederikssund, Denmark), Jørn Brynskov (Gastrounit, Medical Section, Herlev Hospital, University of Copenhagen, Herlev, Denmark), Kent V Haderslev (Department of Medical Gastroenterology, Rigshospitalet, Copenhagen, Denmark) for their contributions to the study and the manuscript.

Declaration of personal interests: AM: None. CJvdW: consultant Abbvie Takeda Mundipharma Janssen Pfizer. MJ: has served on the advisory board of Janssen and Tillotts Pharma, has received research grants for other investigator-driven studies from Takeda, has received consultation fee from Ferring, and has received speaker's fees from MSD, Ferring, and Takeda. EH, AC, SD, TK, ZM, JN, AVH, WVM: None. JS: educational events sponsored by Janssen, MSD, Pfizer and advisory boards for MYLAN, AbbVle. DCB: scientific consultant (advisory boards) AbbVie, MSD, Janssen, Takeda, Boehring Ingelheim, Amgen. CG: Speaker fees: Abbvie, Janssen, Pfizer, Takeda; Advisory Board: Abbvie, Janssen, MSD, Pfizer, Takeda. SN: Abbvie, Takeda, MSD, Amgen, Janssen, Pfizer, Novartis. JR: Speaker fee: Abbvie, MSD, Takeda, Pfizer, Ferring, Falk; Consultancy: Abbvie, Takeda, Hospira, Mundipharma, MSD, Pfizer, GlaxoSK, Amgen; Research grant: Takeda, Abbvie. PB:financial support for research from AbbVie, Mundipharma, Pfizer, Janssen; lecture fees from AbbVie, Takeda, Pfizer and Janssen; advisory board fees from Abbvie, Takeda, Hospira, Janssen, MSD, Mundipharma, Roche, Pfizer, Sandoz and Pentax: None. CE: Grant support/lecture fee/advisory board: Takeda, Janssen Cilag, Pfizer, Abbvie. FH: advisory boards or as speaker for Abbvie, Janssen-Cilag, MSD, Takeda, Celltrion, Teva, Sandoz and Dr Falk. Funding (Grants/Honoraria): Dr Falk, Janssen-Cilag, Abbvie; 
Consulting Fees: Celgene. EL: Research Grant:Takeda, Pfizer; Educational Grant: Abbvie, MSD, Takeda, Janssen ; Speaker Fees: Abbvie, Ferring, MSD, Falk, Takeda, Hospira, Janssen, Pfizer, Celgene; Advisory Board: Abbott, Abbvie, Ferring, MSD, Mitsubishi Pharma, Takeda, Celltrion, Celgene, Hospira, Janssen; Consultant: Abbvie. EM: Grant Tadeda, consultancy fee Janssens en Pfizer: None. AP: Educational grantvan Janssen. SV: Research grant: Takeda, MSD, Abbvie, Pfizer; Consultancy: Abbvie, MSD, Ferring, Takeda, Shire, Janssen, Pfizer Inc, Galapagos, Genentech/ Roche, Celgene, Mundipharma, Eli Lilly, Second Genome, GSK; Speakers fee: AbbVie, MSD, Takeda, Ferring, Dr Falk Pharma, Hospira, Pfizer Incand Tillots. ABGS: consultancy fees from Janssen, Takeda, Abbvie, Pfizer, Medtronic, neopharm and lectures fees from Takeda, Janssen, Rafa laboratories and neopharm. MF: financial support for research: Janssen, Pfizer, Takeda, Consultancy: Abbvie, Boehringer-Ingelheim, Celltrion, Ferring, Janssen, Lilly, Mitsubishi Tanabe, MSD, Pfizer, Takeda; Speakers fee: Abbvie, Amgen, Biogen, Boehringer-Ingelheim, Chiesi, Falk, Ferring, Janssen, Lamepro, Mitsubishi Tanabe, MSD, Pfizer, Takeda, Tramedico, Tillotts, Zeria.

\section{AUTHORSHIP}

Guarantor of the article: Annick Moens.

Author contributions: AM: data acquisition, data analysis and interpretation, statistical analysis, and manuscript writing; MJ, SS, EH, JS, DCB, CG, SN, JR, PB, AC, SD, CE, FH, TK, EL, EM, ZM, JN, $A P, A V H, W V M$ : data acquisition, and manuscript critical revision; CJvdW, SV, MF, ABGS: study concept and design, data acquisition, data analysis and interpretation, manuscript writing. All authors approved the final version of the manuscript.

\section{ORCID}

Annick Moens (iD https://orcid.org/0000-0003-4826-6839

Mette Julsgaard iD https://orcid.org/0000-0003-3070-8950

Carl Eriksson iD https://orcid.org/0000-0002-1046-383X

Edouard Louis iD https://orcid.org/0000-0002-1287-7075

Marc Ferrante iD https://orcid.org/0000-0003-1492-0716

\section{REFERENCES}

1. Mahadevan U, Robinson C, Bernasko N, et al. Inflammatory bowel disease in pregnancy clinical care pathway: a report from the American Gastroenterological Association IBD Parenthood Project Working Group. Gastroenterology. 2019;156:1508-1524

2. van der Woude CJ, Ardizzone S, Bengtson MB, et al. The second European evidenced-based consensus on reproduction and pregnancy in inflammatory bowel disease. J Crohns Colitis. 2015;9:107-124.

3. Vermeire S, Loftus EV, Colombel JF, et al. Long-term efficacy of Vedolizumab for Crohn's disease. J Crohns Colitis. 2017;11: 412-424.

4. Amiot A, Serrero M, Peyrin-Biroulet $L$, et al. Three-year effectiveness and safety of vedolizumab therapy for inflammatory bowel disease: a prospective multi-centre cohort study. Aliment Pharmacol Ther. 2019;50:40-53.

5. Chaparro M, Garre A, Ricart E, et al. Short and long-term effectiveness and safety of vedolizumab in inflammatory bowel disease: results from the ENEIDA registry. Aliment Pharmacol Ther. 2018;48:839-851.

6. Bye WA, Jairath $\mathrm{V}$, Travis S. Systematic review: the safety of vedolizumab for the treatment of inflammatory bowel disease. Aliment Pharmacol Ther. 2017;46:3-15.

7. Mahadevan $\mathrm{U}$, Vermeire $\mathrm{S}$, Lasch $\mathrm{K}$, et al. Vedolizumab exposure in pregnancy: outcomes from clinical studies in inflammatory bowel disease. Aliment Pharmacol Ther. 2017;45:941-950.

8. Moens $\mathrm{A}$, van Hoeve $\mathrm{K}$, Humblet $\mathrm{E}$, et al. Outcome of pregnancies in female patients with inflammatory bowel diseases treated with vedolizumab. J Crohns Colitis 2019;13:12-18.

9. Flanagan E, Gibson PR, Begun J, et al. Letter: vedolizumab drug concentrations in neonates following intrauterine exposure. Aliment Pharmacol Ther. 2018;48:1328-1330.

10. Julsgaard M, Kjeldsen J, Brock B, Baumgart DC. Letter: vedolizumab drug levels in cord and maternal blood in women with inflammatory bowel disease. Aliment Pharmacol Ther. 2018;48: 386-388.

11. Mahadevan $\mathrm{U}$, Vermeire $\mathrm{S}$, Lasch $\mathrm{K}$, et al. Letter: vedolizumab drug levels in cord and maternal blood in women with inflammatory bowel disease-authors' reply. Aliment Pharmacol Ther. 2018;48: 388-389.

12. Julsgaard M, Kjeldsen J, Baumgart DC. Vedolizumab safety in pregnancy and newborn outcomes. Gut. 2017;66:1866-1867.

13. Bar-Gil Shitrit A, Ben Ya'acov A, Livovsky DM, et al. Exposure to vedolizumab in IBD pregnant women appears of low risk for mother and neonate: a first prospective comparison study. Am J Gastroenterol. 2019;114:1172-1175.

14. Seirafi M, de Vroey B, Amiot A, et al. Factors associated with pregnancy outcome in anti-TNF treated women with inflammatory bowel disease. Aliment Pharmacol Ther. 2014;40: 363-373.

15. Kammerlander $\mathrm{H}$, Nielsen J, Knudsen $\mathrm{T}$, Kjeldsen J, Friedman $\mathrm{S}$, Nørgård BM. Anti-TNF- $\alpha$ use during the third trimester of pregnancy in women with moderate-severe inflammatory bowel disease and the risk of preterm birth and low birth weight. Inflamm Bowel Dis. 2017;23:1916-1923.

16. Mahadevan U, Sandborn WJ, Li DK, Hakimian S, Kane S, Corley DA. Pregnancy outcomes in women with inflammatory bowel disease: a large community-based study from Northern California. Gastroenterology. 2007;133:1106-1112.

17. Boyd HA, Basit S, Harpsøe MC, Wohlfahrt J, Jess T. Inflammatory bowel disease and risk of adverse pregnancy outcomes. PLOS ONE. 2015;10:e0129567.

18. Feagan BG, Sandborn WJ, D'Haens G, et al. Induction therapy with the selective interleukin-23 inhibitor risankizumab in patients with moderate-to-severe Crohn's disease: a randomised, doubleblind, placebo-controlled phase 2 study. Lancet. 2017;389(10080): 1699-1709.

19. Zelinkova Z, Berakova K,Podmanicky D, Kadleckova B.Placental MADCAM1 expression and potential consequences for the treatment with vedolizumab during pregnancy. Digestive Disease Week, AGA abstract.

20. Kanis SL, de Lima A, van der Ent C, Rizopoulos D, van der Woude CJ. Anti-TNF levels in cord blood at birth are associated with antiTNF type. J Crohns Colitis. 2018;12:939-947.

21. Beaulieu DB, Ananthakrishnan AN, Martin C, Cohen RD, Kane SV, Mahadevan U. Use of biologic therapy by pregnant women with inflammatory bowel disease does not affect infant response to vaccines. Clin Gastroenterol Hepatol. 2018;16:99-105.

22. Duricova D, Dvorakova E, Hradsky O, et al. Safety of anti-TNFalpha therapy during pregnancy on long-term outcome of exposed 
children: a controlled multicenter observation. Inflamm Bowel Dis. 2019;25:789-796.

23. Bortlik M, Duricova D, Machkova N, et al. Impact of anti-tumor necrosis factor alpha antibodies administered to pregnant women with inflammatory bowel disease on long-term outcome of exposed children. Inflamm Bowel Dis. 2014;20:495-501.

24. Chaparro $M$, Verreth $A$, Lobaton $T$, et al. Long-term safety of in utero exposure to anti-TNF $\alpha$ drugs for the treatment of inflammatory bowel disease: results from the multicenter European TEDDY study. Am J Gastroenterol. 2018;113:396-403.

25. Julsgaard M, Christensen LA, Gibson PR, et al. Concentrations of adalimumab and infliximab in mothers and newborns, and effects on infection. Gastroenterology. 2016;151:110-119.

26. de Lima A, Zelinkova Z, Mulders AG, van der Woude CJ. Preconception care reduces relapse of inflammatory bowel disease during pregnancy. Clin Gastroenterol Hepatol. 2016;14:1285-1292.e1.

27. Pinder M, Lummis K, Selinger CP. Managing inflammatory bowel disease in pregnancy: current perspectives. Clin Exp Gastroenterol. 2016;9:325-335.

\section{SUPPORTING INFORMATION}

Additional supporting information will be found online in the Supporting Information section.

How to cite this article: Moens A, van der Woude CJ, Julsgaard $M$, et al. Pregnancy outcomes in inflammatory bowel disease patients treated with vedolizumab, anti-TNF or conventional therapy: results of the European CONCEIVE study. Aliment Pharmacol Ther. 2020;51:129-138. https://doi.org/10.1111/ apt.15539

\section{APPENDIX 1 \\ THE AUTHORS' COMPLETE LIST OF AFFILIATIONS}

Annick Moens, Séverine Vermeire, Marc Ferrante, Department of Gastroenterology and Hepatology, University Hospitals Leuven, Leuven, Belgium; Department of Chronic Diseases, Metabolism and Ageing, Ku Leuven, Leuven, Belgium; C. Janneke van der Woude, Erasmus MC, Rotterdam, The Netherlands; Mette Julsgaard, Aarhus University Hospital, Aarhus, Denmark; Evelien Humblet, Ziekenhuis Oost-Limburg - Campus Sint-Jan, Genk, Belgium; Juliette Sheridan, St. Vincent's University Hospital, Dublin, Ireland; Daniel C. Baumgart, University of Alberta, Edmonton, AB, Canada; Cyrielle Gilletta De Saint-Joseph, Hôpital Rangueil, Toulouse, France; Stéphane Nancey, CHU Lyon, Lyon, France; Jean-François Rahier, CHU UCL Namur, Université catholique de Louvain, Yvoir, Belgium; Peter Bossuyt, Imeldaziekenhuis, Bonheiden, Belgium; Anneline Cremer, Erasme Hospital, Université Libre de Bruxelles, Brussels, Belgium; Sophie Dewit, Mariaziekenhuis Noord-Limburg, Overpelt, Belgium; Carl Eriksson, Faculty of Medicine and Health Örebro University, Örebro, Sweden; Frank Hoentjen, Radboud University Medical Center, Nijmegen, The Netherlands; Thomas Krause, Opernstrasse, Kassel, Germany; Edouard Louis, CHU Liège, Liège, Belgium; Elisabeth Macken, Universiteit ziekenhuis Antwerpen, UZA, Belgium; Zoran Milenkovic, Military Medical Academy Belgrade, Belgrade, Serbia; Jochen Nijs, SintTrudo Ziekenhuis, Sint-Truiden, Belgium; Annelies Posen, AZ Vesalius, Tongeren, Belgium; Anneleen Van Hootegem, AZ Klina, Brasschaat, Belgium; Wouter Van Moerkercke, AZ Groeninge, Kortrijk, Belgium; Ariella Bar-Gil Shitrit, Shaare Zedek Medical Center, Hebrew University Jerusalem, Jerusalem, Israel. 